\title{
RR Lyrae stars in the outer region of the globular cluster M 3: A shortage of long periods at $r \sim 3.5$ to 6 arcmin?
}

\author{
D. J. Butler ${ }^{\star}$ \\ Max-Planck Institut für Astronomie, Königstuhl 17, 69117 Heidelberg, Germany \\ Received 27 June 2003 / Accepted 31 March 2004

\begin{abstract}
An analysis of the radial distribution of ab-type RR Lyrae star periods in the outer region of the globular cluster M 3 at $r \geq 0.83^{\prime}$ has been performed. That analysis points towards a real shortage of stars with long periods in the radial distance range $3.5^{\prime}$ to $6^{\prime}$ (or about 7 to 12 core radii). A brief discussion is presented. The origin of the phenomenon remains an open question.
\end{abstract}

Key words. stars: variables: RR Lyr - stars: horizontal-branch - stars: evolution - globular clusters: general globular clusters: individual: M3

\section{Introduction}

Accurate distances to resolved stars in the present-day universe are of basic importance for the observational study of the stellar content and formation of individual galaxies over time (e.g. Dolphin et al. 2002; Mackey \& Gilmore 2003; Gratton et al. 2003). By providing a means to evaluate the zero point of the distance scale, RR Lyrae stars, in addition to classical Cepheid stars, provide a powerful constraint on the physics of the formation of the Galaxy and the Local Group of galaxies. For example, for reliable information on stellar masses and their epochs of formation, accurate distances are crucial (e.g. Dolphin et al. 2002). There is however a great need to remove systematic errors both in empirical and theoretical studies of RR Lyrae stars (e.g. see Carretta et al. 2000; Caputo et al. 2000, and references therein). As their periods are more accurately known than other parameters (e.g. magnitudes, colours and amplitudes), the distributions of RR Lyrae star periods in different systems should provide a cleaner test of the theoretical models of RR Lyrae stars. The purpose of the present paper is to use the database of RR Lyrae star periods for M 3 from Corwin \& Carney (2001, referred to hereafter as CC01) and to explore the distribution of periods in the outer region of that star cluster.

There have been past studies of the periods of RR Lyrae stars on different spatial scales. For example, on Galactic scales the mean periods of such stars vary systematically with Galactic latitude (e.g. Fig. 9a in Cseresnjes 2001). Differences in period distributions (e.g., mean period and width) are also known to occur in Galactic sub-systems and satellite galaxies. For example, while period distributions are quite similar

\footnotetext{
* e-mail: butler@mpia.de
}

in some systems (e.g., Sgr and the Large Magellanic Cloud) they are significantly different in others: e.g., compare Sgr with Carina, Leo II or M 15 for instance (Cseresnjes 2001). Do differences occur within any galactic globular clusters? This will be examined in the present paper using the radial distribution of RR Lyrae stars in the outer region of M3, a star cluster with one of the largest RR Lyrae star populations in the Galaxy.

The layout of this short paper is as follows. In Sect. 2, the period data and its radial distribution is presented and analyzed. That is followed by a summary of the result and a basic discussion in Sect. 3.

\section{The adopted sample and radial distributions}

The present study has made use of RR Lyrae star data for M 3 from CC01. Only ab-type stars at $r \geq 0.83^{\prime}$ have been considered in order to have an essentially complete sample of periods: based on an inspection of the data, completeness at $0.83^{\prime} \leq r \leq$ $1.7^{\prime}$ is about $80 \%$ and is $100 \%$ at larger radii. Periods are precise to much better than $0.1 \%$, and the spatial coordinates of isolated stars are precise to typically 0.15" (Bakos et al. 2000).

Figures $1 \mathrm{a}$ and $\mathrm{b}$ plot the radial distribution of periods and their dispersion in four annular bins respectively. Each annular region and its star count is listed in Table 1. In order to be insensitive to outliers in the data, a so-called bootstrap technique has been used to calculate the dispersion in each radial bin and the associated uncertainty: $80 \%$ of the stars in each radial bin were randomly selected, and the dispersion was recalculated. For each radial bin, this procedure was repeated several hundred times; the mean of the resultant set of values was taken as the period dispersion while the standard deviation 

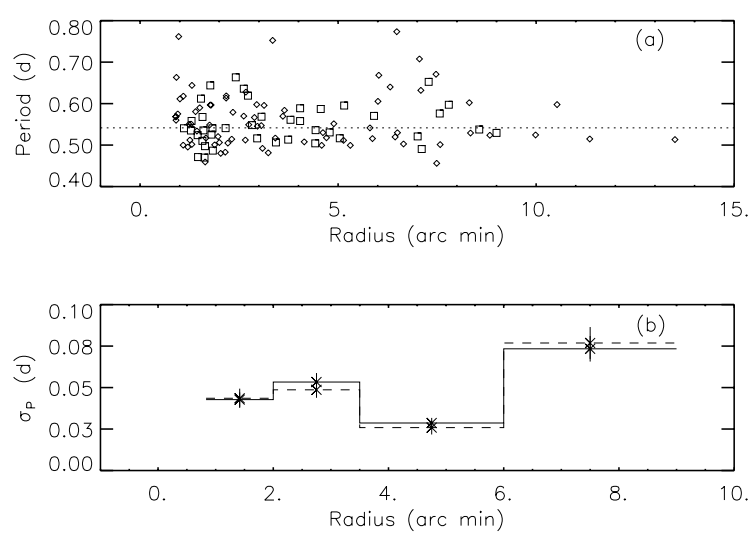

Fig. 1. a) Radial distribution of the periods of Blazhko stars (squares), and non-Blazhko ab-type stars (diamonds) at $r \geq 0.83^{\prime}$. The shortdash line marks the median period of the ensemble data. b) Standard deviation of periods in annular bins as a function of radial position for Blazhko plus non-Blazhko stars (solid) and non-Blazhko stars (dashed). See the text in Sect. 2 for further information.

Table 1. Numbers of RR Lyrae stars in different annular regions in M3. Columns (2) to (3) have been produced using RR Lyrae star classifications from CC01/Bakos et al. (2000). See text in Sect. 2 for further information.

\begin{tabular}{lll}
\hline \hline Annulus & $N_{\mathrm{RRab}^{a}}{ }^{a}$ & $N_{\text {non-Blazhko }}$ \\
$(1)$ & $(2)$ & $(3)$ \\
\hline $0.83^{\prime}-2.00^{\prime}$ & 40 & 25 \\
$2.00^{\prime}-3.50^{\prime}$ & 29 & 21 \\
$3.50^{\prime}-6.00^{\prime}$ & 22 & 11 \\
$6.00^{\prime}-9.00^{\prime}$ & 21 & 15 \\
\hline
\end{tabular}

${ }^{a}$ Non-Blazhko and Blazhko ab-type RRLyrae stars.

was taken as the (random) dispersion uncertainty. To determine how much the uncertainty estimates depend on the percentage of stars selected, the percentage selected was varied from $70 \%$ to $90 \%$ : a similar mean dispersion value was obtained and only a slight change in uncertainty was found.

For an assessment of the probability that the measured dispersion at $3.5^{\prime}$ to $6^{\prime}$ could have occurred by chance ${ }^{1}$, the following method has been applied: if a radial bin contained $\mathrm{N}$ stars, then $\mathrm{N}$ stars were picked randomly from the whole sample, and the standard deviation was calculated. This was repeated a significant number of times (10000) for each radial bin, thus providing a separate histogram of occurrences for each bin. The probability of occurrence of the measured dispersion values is less than $0.01 \%$ for either the set of non-Blazhko ${ }^{2}$ ab-type star

${ }^{1}$ It is noted that a Kolmogorov-Smirnov (K-S) test, which is sometimes used to test the significance of the difference between two distributions is not adequate for the data, unlike the adopted test: the sensitivity of the K-S test is best when the determined probability statistic does not depend on the tails of the distributions used but rather the central region where number statistics is higher.

2 The Blazhko effect is common in ab-type RR Lyrae stars; rare in c-type RR Lyrae stars; and is characterized by cycle-to-cycle variations in luminosity and radial velocity curves (i.e. influencing amplitude).

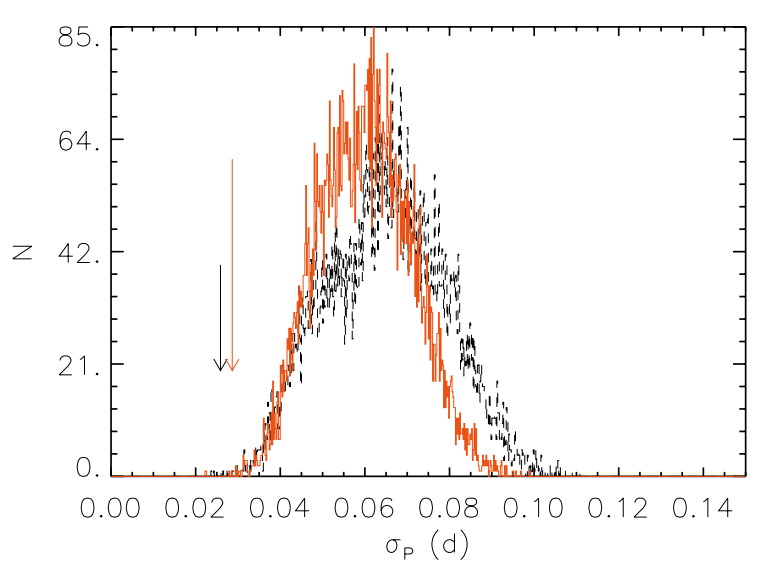

Fig. 2. Frequency of occurrence of $\sigma_{\mathrm{P}}$ at $r=3.5^{\prime}$ to $6^{\prime}$ for non-Blazhko ab-type stars (dashed) as well as Blazhko plus non-Blazhko ab-type stars (solid). Arrows indicate the measured dispersion in the same radial range for both the former case (long) and the latter case (short).

periods or the ensemble data. The probability of a value less than or equal to the measured values is $0.03 \%$ and $0.07 \%$ respectively. Accordingly, this points towards a shortage of long period stars at $3.5^{\prime}$ to $6^{\prime}$ in M 3, regardless of whether Blazhko stars are included in the analysis or not. Histograms for the radial bin at $3.5^{\prime}$ to $6^{\prime}$ are shown in Fig. 2. Vertical arrows indicate the measured dispersion associated with each histogram.

For a check of the effect of bin size and bin location, bin sizes at $r \geq 0.83^{\prime}$ have been scaled simultaneously by up to $5 \%$. The result is a small change in dispersion values and negligible change in error bar sizes - importantly, the probability that the radial minimum in dispersion values occurred by chance is still low, being less than $0.1 \%$. As another check, the same data was binned at intervals of $1^{\prime}$ and interestingly, even with reduced number statistics, a significant radial minimum occurred in the bin at about $5^{\prime}$ to $6^{\prime}$.

\section{Result and discussion}

In the previous section it was shown clearly that there is evidence for a possible shortage of long period stars of the type $\mathrm{ab}$ in the outer region of M3. It relies on the high precision and accuracy of periods even in the innermost regions, unlike mean colours and amplitudes that may be affected by systematic photometric uncertainties due to crowding. It is stated here as only a possible shortage because although random errors are assessed well by the boot-strap technique applied earlier, it is not possible to rule out the possibility of a systematic error arising from the finite size of the RR Lyrae star population. With this a caveat, it is concluded that the general trend for the dispersion of period values is a drop from the inner region sampled $\left(0.83^{\prime}\right.$ to $\left.3.5^{\prime}\right)$ to smaller values in an intermediate radial region $\left(3.5^{\prime}\right.$ to $\left.6^{\prime}\right)$, and an increase again toward the outer region sampled (beyond about $6^{\prime}$ ). Beyond a radial distance of about $9^{\prime}$, there is little information due to a decreasing surface density of stars.

Due to the potential importance of the result in the context of single star evolution in star clusters, an important parameter worth speculating about briefly is chemical composition. 
Both observationally (e.g. Sandage 1982) and theoretically (e.g. Bono et al. 2001) it is known that there is a correlation between period, heavy element abundance and $K$-band magnitude, i.e. the PKZ relation ${ }^{3}$. Thus, if taken at face value, the apparent shortage of long periods should be reflected in $K$-band magnitudes and/or Z. For example, applying the tight PKZ relation (e.g. Eq. (3) of Bono et al. 2001), one finds that for $M_{K}=-0.3$ mag at a mid-way period of $0.5 \mathrm{~d}$, a period increase of $0.05 \mathrm{~d}$ would correspond to $\Delta \log Z \sim 0.6 \mathrm{dex}$. The current census of $[\mathrm{Fe} / \mathrm{H}]$ values is however too incomplete to warrant an examination of its radial distribution at the present time.

Another issue worth mentioning is the spatial distribution of RR Lyrae stars in M3. A Kolmogorov-Smirnov test of the cumulative radial distribution of the RR Lyrae stars and red giant branch (RGB) stars, using RGB data from Ferraro et al. (1997; and references therein), indicates a probability of $82 \%$ that they are from the same parent populations (proof of the null-hypothesis). That suggests that the dynamical histories of the RR Lyrae star and RGB star populations in M 3 are similar in a statistical sense over the range of radial distances examined in the present paper. Consequently, if there is some form of selection mechanism that affects RR Lyrae stars, and if that mechanism is linked to the dynamics of cluster evolution, its effect on RGB stars, if any, may vary with radial distance in the cluster.

Lastly, although there is no supporting empirical evidence, it is of academic interest to sketch very briefly one intriguing potential selection mechanism; that is the issue of gas giant planets or brown dwarfs that may be interacting with stars evolving along the RGB (Soker 1998). It is believed that the mass loss rate of RGB stars would be increased by the release of energy and angular momentum on entering the envelope of the RGB star. In the reported scenario, increasing mass loss would cause a star to become bluer as it starts towards the HB (Soker 1998). Speculatively, one could imagine that this might preferentially select short RR Lyrae star periods, and that the efficiency of such a mechanism might well vary significantly with radial distance in a star cluster.

Acknowledgements. An anonymous referee is thanked for pertinent comments that helped the author to improve the quality of this paper. D. J. Butler acknowledges the support of the European research and training network on "Adaptive Optics for Extremely Large Telescopes" under contract HPRN-CT-2000-00147.

\section{References}

Bakos, G. A., Benko, J. M., \& Jurcsik, J. 2000, AcA, 50, 221

Bono, G., Caputo, F., Castellani, V., Marconi, M., \& Storm, J. 2001, MNRAS, 326, 1183

Caputo, F., Castellani, V., Marconi, M., \& Ripepi, V. 2000, MNRAS, 316,819

Carretta, E., Gratton, R. G., \& Clementini, G. 2000, MNRAS, 316, 721

Corwin, M. T., \& Carney, B. W. 2001, AJ, 122, 3183

Cseresnjes, P. 2001, A\&A, 375, 909

Dolphin, A., Saha, A., Claver, J., et al. 2002, AJ, 123, 3154

Ferraro, F. R., Paltrinieri, B., Fusi Pecci, F., et al. 1997, A\&A, 324, 915

Gratton, R. G., Bragaglia, A., Carretta, E., et al. 2003, A\&A, 408, 529

Mackey, A. D., \& Gilmore, G. F. 2003, MNRAS, 345, 747

Roberts, M., \& Sandage, A. 1955, AJ, 60, 185

Sandage, A. 1982, ApJ, 252, 553

Soker, N. 1998, AJ, 116, 1308

\footnotetext{
${ }^{3}$ The apparent shortage of long periods at $r=3.5-6^{\prime}$ seems to be reflected in the $B$ - and $V$-band amplitude data from CC01. It is expected because of a known correlation between optical amplitudes and periods in M3 (e.g., Roberts \& Sandage 1955, Fig. 6; CC01, Fig. 9). Herein an amplitude analysis is excluded because of likely systematic effects caused by light curve scatter and/or stellar crowding.
} 